\title{
An Effective Method for Illumination-Invariant Representation of Color Images
}

\author{
Takahiko Horiuchi ${ }^{1}$, Abdelhameed Ibrahim ${ }^{1,2}$, \\ Hideki Kadoi ${ }^{1}$, and Shoji Tominaga ${ }^{1}$ \\ 1 Graduate School of Advanced Integration Science, Chiba University, \\ Yayoi-cho 1-33, Inage-ku, Chiba 263-8522, Japan \\ 2 Faculty of Engineering, Mansoura University, Mansoura, Egypt \\ \{horiuchi, shoji\}@faculty.chiba-u.jp
}

\begin{abstract}
This paper proposes a method for illumination-invariant representation of natural color images. The invariant representation is derived, not using spectral reflectance, but using only RGB camera outputs. We suppose that the materials of target objects are composed of dielectric or metal, and the surfaces include illumination effects such as highlight, gloss, or specularity. We preset the procedure for realizing the invariant representation in three steps: (1) detection of specular highlight, (2) illumination color estimation, and (3) invariant representation for reflectance color. The performance of the proposed method is examined in experiments using real-world objects including metals and dielectrics in detail. The limitation of the method is also discussed. Finally, the proposed representation is applied to the edge detection problem of natural color images.
\end{abstract}

Keywords: Illumination-invariant, color image, highlight detection, spectral reflectance estimation.

\section{Introduction}

Color information observed from object surfaces provides crucial information in computer vision and image analysis which include the essential problems of feature detection, image segmentation, object recognition, and image retrieval. However, in real-world applications there are various illumination factors that can affect the appearance of color images captured from object surfaces. The captured images do not only depend on surface-spectral reflectances and illuminant spectrum, but also include reflection effects such as shading, gloss, and highlight, which mainly depend on illumination geometries and surface materials. Therefore, image representations invariant to shading, shadow, lighting, and specularity have been proposed for color images [1-4] and for spectral images [5, 6] so far in several ways. However, most of the illumination-invariant methods used the standard dichromatic reflection model by Shafer [7]. This model is valid for such limited materials of inhomegeneous dielectrics as plastics and paints. It should be noted that there are metallic objects in real-world scenes, which cannot be described by the standard dichromatic reflection model.

A. Fusiello et al. (Eds.): ECCV 2012 Ws/Demos, Part II, LNCS 7584, pp. 401-410, 2012.

(C) Springer-Verlag Berlin Heidelberg 2012 
In our previous studies, we proposed an invariant representation for a variety of real-world objects including metals and dielectrics [8]. We showed that the normalized surface spectral reflectance by the minimum reflectance was invariant to illumination effects and the representation was effective to spectral imaging applications [9]. However, the method requires an expensive and special measurement device such as a spectral imaging system and a standard white reference. Furthermore, long measurement time is necessary due to acquisition of the high-dimensional spectral data.

The present paper proposes a method for illumination-invariant representation of natural color images captured by an off-the-shelf RGB camera, and its application to image processing. The illumination-invariant representation of spectral reflectance is not available directly for this problem because the illuminant spectrum is unknown. We derive the invariant representation by not using spectral reflectance, but using only three sensor outputs. Estimation of illumination (light source) color is required separately. We suppose that the materials of target objects are composed of dielectric or metal, and the surfaces include illumination effects such as highlight, gloss, or specularity. We propose an algorithm for realizing the invariant representation for color images in three steps: (1) detection of specular highlight, (2) illumination color estimation, and (3) invariant representation for object color.

\section{Invariant Representation for Spectral Reflectances}

The illuminant-invariant representation for surface-spectral reflectances can be derived from the standard dichromatic reflection model for dielectric and the extended reflection model for metal [8]. The spectral reflectances of object surfaces in a scene are observed by specific devices such as a spectral imaging system, a standard white reference, and a spectroradio/photometer. The typical imaging system consists of a liquid crystal tunable (LCT) filter and a monochrome camera. Then it is found that the normalized surface-spectral reflectances by the minimum reflectance among the observed spectral reflectances are invariant to highlight, shading, surface geometry, and illumination intensity. The invariant representation for spectral reflectance is described as

$$
S^{\prime}(\theta, \lambda)=\frac{S(\theta, \lambda)-\min \{S(\theta, \lambda)\}}{\sqrt{\int_{400}^{700}(S(\theta, \lambda)-\min \{S(\theta, \lambda)\})^{2} d \lambda}},
$$

where $S(\theta, \lambda)$ is the spectral reflectance function of the wavelength $\lambda$, observed in the range of a visible wavelength [400, $700 \mathrm{~nm}]$, and the geometric parameters $\theta$, including the direction angles of the viewing angle and the phase angle. For precise spectral representation, spectral images are usually captured at equal interval of $5 \mathrm{~nm}$ or $10 \mathrm{~nm}$ in the visible range. The imaging system using a LCT filter takes more than 150 second to capture 31-band images at $10 \mathrm{~nm}$ interval. Therefore, use of expensive devices and long measurement time are bottleneck towards practical use. 


\section{Principle of Invariant Representation for Color Images}

\subsection{Dichromatic Reflection Models}

The standard dichromatic reflection model suggests that light reflected from the surface of an inhomogeneous dielectric object is composed of interface reflection and body reflection.

Let $\mathbf{C}_{S}=\left[C_{S}^{(R)}, C_{S}^{(G)}, C_{S}^{(B)}\right]^{T}$ be a 3-D column vector representing the red, green, and blue sensor responses of a color imaging system. The sensor outputs can be expressed as

$$
\mathbf{C}_{S}=m_{I} \mathbf{C}_{I}+m_{B} \mathbf{C}_{B}
$$

where the vectors $\mathbf{C}_{I}$ and $\mathbf{C}_{B}$ are the color vectors representing, respectively, the interface and body reflection components of the color signal reflected from the object. The weights $m_{I}$ and $m_{B}$ are the geometric scale factors.

The standard model incorporates the neutral interface reflection (NIR) assumption which states that the interface reflection component is coincident with the color of light source. This allows Eq.(2) to be written as

$$
\mathbf{C}_{S}=m_{I} \mathbf{C}_{L}+m_{B} \mathbf{C}_{B}
$$

where $\mathbf{C}_{L}$ is a constant color vector of light source. It is shown that this ref lection model is valid for a variety of natural and artificial dielectric objects includi ng plastic and paint.

Metal is a homogeneous material that indicates essentially different reflection properties from the dielectric materials. It consists of only interface reflection with the Fresnel reflectance. So, if the surface is shiny and stainless, the body reflection component is negligibly small. A sharp specular highlight is observed only at the viewing angle of the mirrored direction. Thus the surface reflection depends on the incident angle of illumination.

Tominaga [10] shows that the spectral reflection of metal surface can be approximated by a linear combination of only two interface reflection components. This type of surface reflection is called the extended dichromatic reflection model. The color model can be expressed as

$$
\mathbf{C}_{S}=m_{I 1} \mathbf{C}_{I 1}+m_{I 2} \mathbf{C}_{I 2}
$$

where the first term in the right hand side corresponds to the specular color component at the normal incident and the second corresponds to the grazing color component at the horizontal incident. It is noted that surface-spectral reflectance is whitened at the grazing angle. Therefore, the observed color vector can be expressed in a linear combination of the metal color at the normal incident and the light source color $\mathbf{C}_{L}$ as follows

$$
\mathbf{C}_{S}=m_{I 1} \mathbf{C}_{I 1}+m_{I 2} \mathbf{C}_{L}
$$




\subsection{Illuminant-Invariant Representation}

We briefly explain an illumination-invariant representation, which is limited to the standard dichromatic reflection model for inhomogeneous dielectric objects, and then extend the invariant representation for all materials including dielectric and metal. We focus on the 3-D RGB-space. Let us consider that illumination is not white but colored without $C_{L}^{(R)}=C_{L}^{(G)}=C_{L}^{(B)}$. Let $\hat{\mathbf{C}}_{L}$ be an estimate of the illumination color vector obtained in a separate way. Then the influence of illumination color is reduced from the sensor outputs as

$$
\hat{\mathbf{C}}_{S}=\mathbf{C}_{S} / \hat{\mathbf{C}}_{L},
$$

where the division "/" is done in element-wise operation. The above equation means the sensor output for the same object surface under a standard white illumination and the transformed color $\hat{\mathbf{C}}_{S}$ is named "reflectance color" in this paper. With a reliable estimate $\hat{\mathbf{C}}_{L}$, the standard reflection model in Eq.(3) allows Eq.(6) to be written in the following form

$$
\hat{\mathbf{C}}_{S}=m_{I} \mathbf{I}+m_{B}\left(\mathbf{C}_{B} / \hat{\mathbf{C}}_{L}\right),
$$

where $\mathbf{I}$ is a unit vector with $[1 / 3,1 / 3,1 / 3]^{T}$. Thus the sensor vector $\hat{\mathbf{C}}_{S}$ has a constant specular reflection component. Let $\hat{\mathbf{C}}_{B}=\mathbf{C}_{B} / \hat{\mathbf{C}}_{L}$ be the body color normalized by the light source color, which means the object color observed under white illumination (canonical color). Then, subtraction of one color component from another component provides a representation independent of the specular reflection component as follows

$$
\hat{C}_{S}^{(R)}-\hat{C}_{S}^{(G)}=\left(m_{I}+m_{B} \hat{C}_{B}^{(R)}\right)-\left(m_{I}+m_{B} \hat{C}_{B}^{(G)}\right)=m_{B}\left(\hat{C}_{B}^{(R)}-\hat{C}_{B}^{(G)}\right),
$$

where the geometric weighting coefficient $m_{I}$ is eliminated. In this case, the ratio of two subtractions between color components can be illumination-invariant, that is, invariant to highlight, shading, and surface geometry by eliminating the remaining weighting coefficient $m_{B}$.

$$
\frac{\hat{C}_{S}^{(R)}-\hat{C}_{S}^{(G)}}{\hat{C}_{S}^{(G)}-\hat{C}_{S}^{(B)}}=\frac{m_{B}\left(\hat{C}_{B}^{(R)}-\hat{C}_{B}^{(G)}\right)}{m_{B}\left(\hat{C}_{B}^{(G)}-\hat{C}_{B}^{(B)}\right)}=\frac{\hat{C}_{B}^{(R)}-\hat{C}_{B}^{(G)}}{\hat{C}_{B}^{(G)}-\hat{C}_{B}^{(B)}} .
$$

Next, we consider the image sensor outputs for a metal object. In a similar way, the normalization of Eq.(6) is applied to the extended reflection model Eq.(5). We obtain

$$
\hat{\mathbf{C}}_{S}=m_{I 1}\left(\mathbf{C}_{I 1} / \hat{\mathbf{C}}_{L}\right)+m_{I 2} \mathbf{I} .
$$

Note that, Eq.(10) for the extended reflection model is the mathematically same fashion as Eq. (77) for the standard model, although the two equations have different physical meaning. Therefore, we derive a unified invariant representation for all materials including dielectric and metal. In fact, an invariant equation for metal is derived from Eq.(10) with $\hat{\mathbf{C}}_{I 1}=\mathbf{C}_{I 1} / \hat{\mathbf{C}}_{L}$ as

$$
\frac{\hat{C}_{S}^{(R)}-\hat{C}_{S}^{(G)}}{\hat{C}_{S}^{(G)}-\hat{C}_{S}^{(B)}}=\frac{m_{I 1}\left(\hat{C}_{I 1}^{(R)}+m_{I 2}\right)-\left(m_{I 1} \hat{C}_{I 1}^{(G)}+m_{I 2}\right)}{m_{I 1}\left(\hat{C}_{I 1}^{(G)}+m_{I 2}\right)-\left(m_{I 1} \hat{C}_{I 1}^{(B)}+m_{I 2}\right)}=\frac{\hat{C}_{I 1}^{(R)}-\hat{C}_{I 1}^{(G)}}{\hat{C}_{I 1}^{(G)}-\hat{C}_{I 1}^{(B)}} .
$$


where the geometric weighting coefficients $m_{I 1}$ and $m_{I 2}$ are eliminated. We note that the operation of Eq.(11) results in an equivalent operation to Eq.(9), that is invariant to highlights, shading, and geometries.

\section{Procedure for Invariant Representation}

\subsection{Detection of Specular Highlight}

It is well-known that specular highlight is useful for illuminant estimation because the spectral reflectance of the interface reflection component is constant over the visible range and the color vector is coincident with illumination color as in Eq.(3). So far, several methods were presented for detecting bright specular highlights on the observed object surfaces (e.g., see 11]). However, note that this illuminant estimation method is available for dielectrics at any incidence angle of illumination, but available for metals at a limited angle with incidence near the grazing angle.

In this paper, we use a highlight detection method based on variable luminance thresholding. First, we calculate the luminance value from the captured RGB image at every pixel and make a luminance histogram for the entire image. Second, highlight candidates are extracted using a single threshold value, which corresponds to the luminance at a clear valley in the distribution curve of the luminance histogram. Third, the extracted candidate areas are labeled based on connectivity of pixels. If the connected pixel count is small, the area is eliminated. Then the connected areas with enough pixel counts are reserved as the highlight areas. Fourth, each highlight area is narrowed by increasing the threshold value, in order to determine precisely the highlight area. The above process is repeated for the respective target regions.

It should be noted that the detection algorithm is very simple, although the detection accuracy is not so high. However, the detection of a few high lights is enough for estimating the illumination color. Therefore, an accurate detection algorithm is not necessary for this purpose. We verified that this detection algorithm was effective empirically.

\subsection{Illumination Color Estimation}

Considering the dichromatic reflection property, the image data in each detected highlight area of object surfaces are projected onto a 2-D space using two principal components by

$$
\left[\begin{array}{l}
\boldsymbol{\xi}_{1} \\
\boldsymbol{\xi}_{2}
\end{array}\right]=\left[\begin{array}{c}
\mathbf{V}_{1}^{T} \\
\mathbf{V}_{2}^{T}
\end{array}\right] \mathbf{C}_{S}
$$

where $\mathbf{V}_{1}$ and $\mathbf{V}_{2}$ denote the first and second principal component vectors, which are computed from the image data set $\left\{\mathbf{C}_{S}\right\}$ belonging to the highlight area. A histogram of the projected 2-D image data $\left(\xi_{1}, \xi_{2}\right)$ consists of two clusters for the specular reflection component and the diffuse reflection component according to the dichromatic reflection model. Then, the cluster of specular reflection 
component indicating the light-source color is extracted to estimate illuminant. Note that the histogram is divided into two linear clusters. Then, the specular cluster corresponds to highlight pixels by the specular reflection.

If the specular highlight area is much brighter than the matte area, the linear highlight cluster is much longer than the linear cluster of the diffuse component, so that the highlight cluster is regarded as a single linear cluster. In this case, the first principal component vector of the highlight cluster corresponds to the directional vector representing the illumination. The illumination color vector can be recovered by extracting the first principal component vector of the highlight cluster and transforming it inversely into the original 3-D color space.

Let $\left(\hat{\xi}_{1}, \hat{\xi}_{2}\right)$ be the directional vector extracted from the specular highlight cluster. Then the illumination color vector $\hat{\mathbf{C}}_{L}$ is estimated as follows:

$$
\hat{\mathbf{C}}_{L}=\hat{\xi}_{1} \mathbf{V}_{1}+\hat{\xi}_{2} \mathbf{V}_{2} \text {. }
$$

If the object color is similar to the illumination color, the estimation accuracy may decrease.

\subsection{Invariant Representation for Reflectance Color}

We can generalize the invariant expressions in Eq.(11) to a stable expression based on the subtraction between any color component and the minimal component. The generalized representation for the reflectance color $\hat{\mathbf{C}}_{S}$ is given as

$$
\hat{C}_{S}^{\prime(i)}=\frac{\hat{C}_{S}^{(i)}-\min \left\{\hat{C}_{S}^{(R)}, \hat{C}_{S}^{(G)}, \hat{C}_{S}^{(B)}\right\}}{\sqrt{\sum_{j \in\{R, G, B\}}\left(\hat{C}_{S}^{(j)}-\min \left\{\hat{C}_{S}^{(R)}, \hat{C}_{S}^{(G)}, \hat{C}_{S}^{(B)}\right\}\right)^{2}}} .
$$

This representation is effective in the sense of measurement and computation time.

\section{Experiments}

Figure1(a) shows an experimental scene, including a metal object of copper and two dielectric objects of ceramic (cup) and plastic (frog), which are illuminated with the light source of an incandescent lamp. The color images were captured using a Canon 5D Mark II digital camera. We assume that the specular highlight pixels are not clipped. The image with $445 \times 273$ pixels in Fig 1. (a) was analyzed.

First, four areas surrounded by yellow rectangles in Fig 1.(a) are the detection results of specular highlight areas. Second, the illumination color vector $\hat{\mathbf{C}}_{L}$ was estimated at each decected highlight region by the principal component analysis of highlight clusters in 2D histogram of these areas, as shown in Sec 4.2 Then the estimated color vectors were averaged. The estimated illumination color vector was then compared with a direct measurement using a standard white reference. A fairly good coincidence between the estimate and the measurement was obtained with the RMSE of 0.044. Figs. 1(b) and 1(c) show the surface 
color image corrected from the observed image using the white reference and the surface color image corrected using the estimated illumination color vector, respectively. These corrections were done by element-wise division as indicated in Eq.(6). These results suggest that the influences of illumination color are reduced in the surface color images corrected using the estimated illumination color vector $\hat{\mathbf{C}}_{L}$.
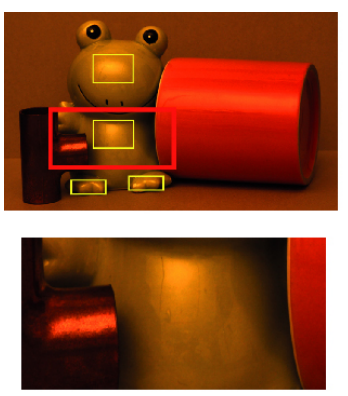

(a)
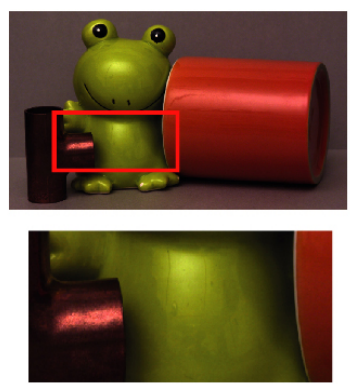

(b)
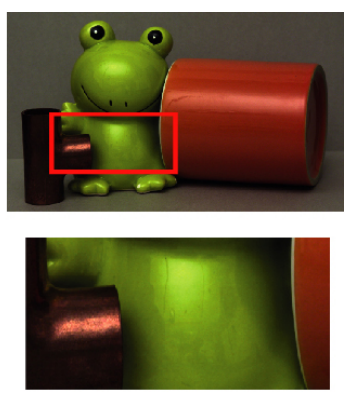

(c)

Fig. 1. Color image correction: (a) observed color image and detected highlight regions; (b) corrected image using the white reference; (c) corrected image using the estimated illumination color vector

Figure 2(a) depicts a 3-D view of a single channel image ( $\mathrm{R}$ channel) for the small rectangular area including metal and dielectrics. That is, the figure represents the $\mathrm{R}$ component of reflectance. Note that the reflectance color image has the influences of shading and specularity. In contrast, 2(b) shows the 3-D view of the illumination-invariant representation for the same part at the $\mathrm{R}$ channel. Note that all shadows and highlights disappear from the original color image, so that the invariant image is composed of only the reflection component inherent to each object surface.

It is obvious that the proposed invariant method for color images is simpler and faster than the invariant method for spectral images in Ref. 8]. However, the proposed method based on color images has a limitation. It is the problem of metamer. For instance, our invariant representation may become the same between two object surfaces, even if those are different spectrally. Figure 31(a) shows a test scene, which has two plastic objects with similar object colors but slightly different surface spectral reflectances as shown in Fig. 3(b). Figures 4(a) and 4(b) show the invariant representations using the spectral image and the color image, respectively. Figures 4(c) and 4(d) shows their region classification results using the normalized cut approximated by the Nyström method. Two objects are segregated in Fig. 4(c) and not segregated in Fig. 4(d), although illumination effects disappear from both objects. An additional processing may be required for such an object classification. 


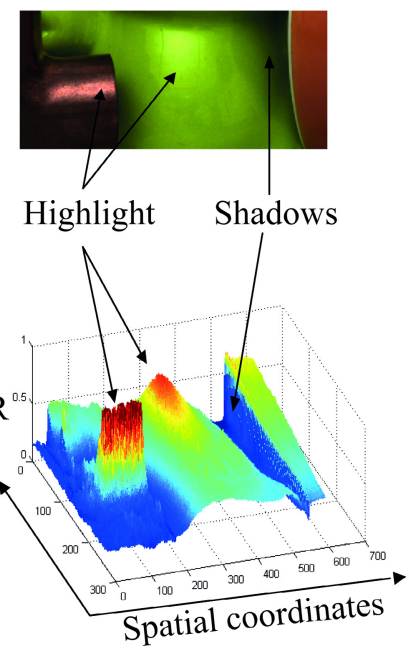

(a)

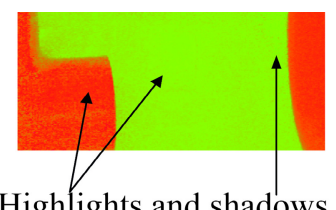

Highlights and shadows

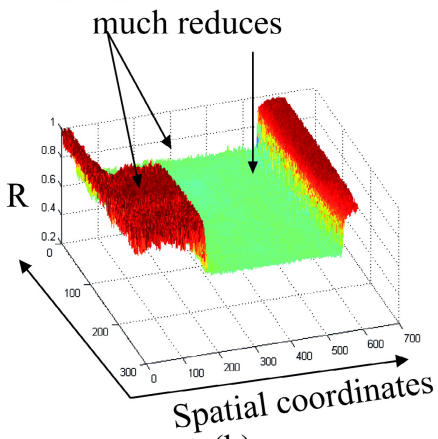

(b)

Fig. 2. Illumination-invariant evaluation for a natural color image. (a) 3-D view of the component image at the R-channel; (b) 3-D view of the illumiantion-invariant representation for the same part at the R-channel.

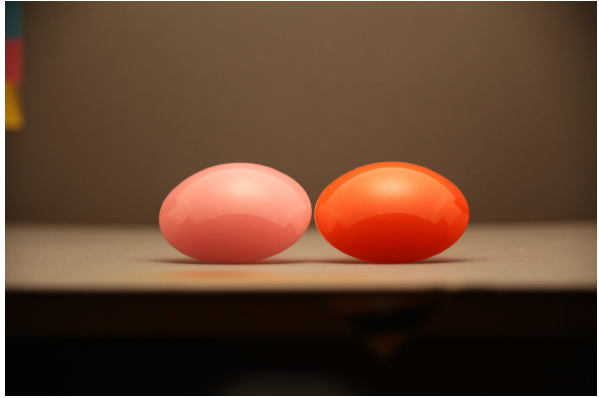

(a)

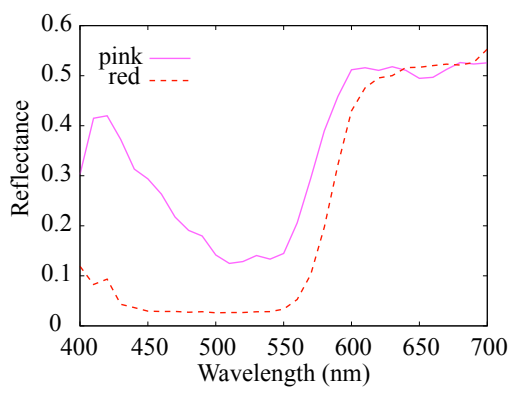

(b)

Fig. 3. A test scene. (a) original color image. (b) Surface-spectral reflectances for two plastic objects in (a).

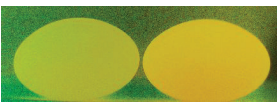

(a)

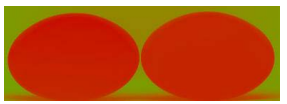

(b)

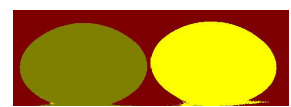

(c)

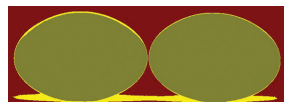

(d)

Fig. 4. Experimental results for Fig. 3(a). (a) Invariant representation from the spectral image. (b) Invariant representation from the color image. (c) Object classification result from the spectral image. (d) Object classification result from the color image. 


\section{Applications to Edge Detection}

Let us consider the edge detection problem of natural color images based on the proposed illumination-invariant representation. We calculate the gray-scale edge strength using the Gaussian color model 22] from the normalized color values and the illumination-invariant representation to show the effectiveness of the reflectance color images in reducing the illumination conditions. A white light source is assumed in the present experiment. Figure 5(a) shows a color test scene used in Ref. [3]. Figure 5(b) depicts the gray-scale edge strength of the original tested part. The binary edges are automatically calculated by Otsu's method from the gray-scale image. The upper images and lower images indicate gray-scale images and binary images, respectively. The result contains many shadows and highlights effects. Figure 5(c) shows the gray-scale edges of the proposed representation. The edges clearly separate different materials of metal and dielectric. Thus the proposed representation is independent of the illumination effects of highlight and shadow, and the geometry of object shape. To confirm the usefulness of the proposed method, the first results using the photometric quasi-invariant method [3] are shown in Fig. [5(d) with binary edges. The second result using the color invariance method [2] are shown in Fig. 55(e).

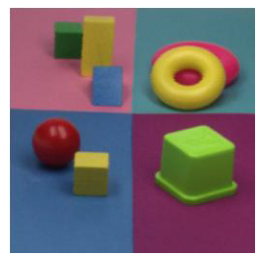

(a)
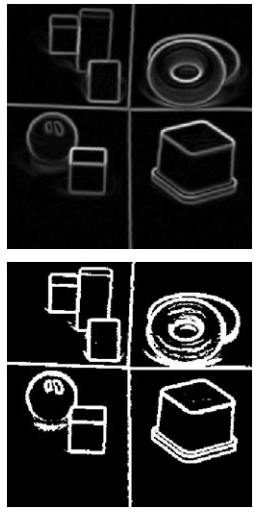

(b)
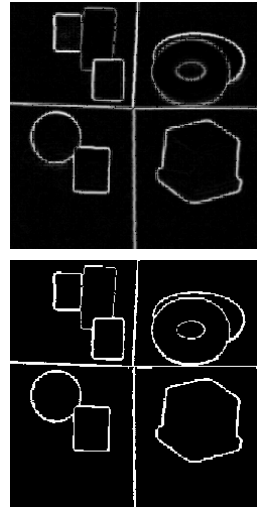

(c)
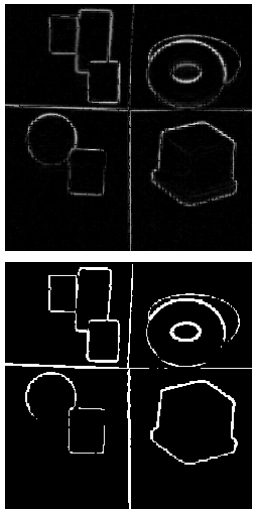

(d)
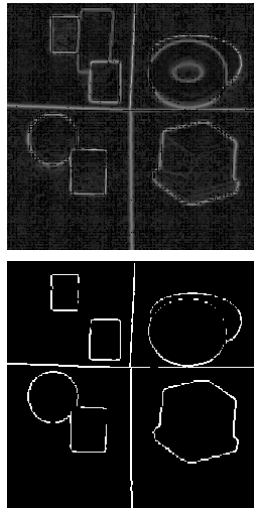

(e)

Fig. 5. Edge detection evaluation; (a) a color test scene [3], (b) all edges, (c) proposed invariant method (d) quasi-invariant method [3], (e) color invariant method [2] 


\section{Conclusion}

This paper has proposed a method for illumination-invariant representation of natural color images. The target objects were supposed to be dielectric and metal, and the surfaces included illumination effects such as highlight, gloss, or specularity. The procedure for realizing the invariant representation consisted of three steps: (1) detection of specular highlight, (2) illumination color estimation, and (3) invariant representation for reflectance color. The performance of the proposed method was examined in experiments using real-world objects including metals and dielectrics in detail. The results showed that the proposed representation was invariant to highlight, shadow, object surface geometry, and illumination intensity. Moreover, we applied the invariant representation to the edge detection problem successfully.

We also explore as a future work an objective evaluation of the proposed invariant representation for complex scenes including noise.

\section{References}

1. Burghouts, G.J., Geusebroek, J.-M.: Performance Evaluation of Local Colour Invariants. Computer Vision and Image Understanding 113, 48-62 (2009)

2. Geusebroek, J.M., Boomgard, R.V., Smeulders, A.W.M., Geerts, H.: Color Invariance. IEEE Trans. Pattern Analysis and Machine Intelligence 23, 1338-1350 (2001)

3. van de Weijer, J., Gevers, T., Geusebroek, J.M.: Edge and Corner Detection by Photometric Quasi-Invariants. IEEE Trans. Pattern Analysis and Machine Intelligence 27, 625-630 (2005)

4. van de Weijer, J., Gevers, T., Smeulders, A.W.M.: Robust Photometric Invariant Features from the Color Tensor. IEEE Trans. Image Processing 15, 118-127 (2006)

5. Stokman, H.M.G., Gevers, T.: Detection and Classification of Hyper-spectral Edge. In: Proc. 10th British Machine Vision Conference, pp. 643-651 (1999)

6. Montoliu, R., Pla, F., Klaren, A.K.: Multispectral invariants. Technical Report, DLSI, Universitat Jaume I (2004)

7. Shafer, S.A.: Using Color to Separate Reflection Components. Color Research and Applications 10, 210-218 (1985)

8. Ibrahim, A., Tominaga, S., Horiuchi, T.: A spectral Invariant Representation of Spectral Reflectance. Optical Review 18, 231-236 (2011)

9. Ibrahim, A., Tominaga, S., Horiuchi, T.: Invariant Representation for Spectral Reflectance Images and Its Application. EURASIP Journal on Image and Video Processing 2011 (2011)

10. Tominaga, S.: Dichromatic Reflection Models for a Variety of Materials. Color Research and Applications 19, 277-285 (1994)

11. Imai, Y., Kato, Y., Kadoi, H., Horiuchi, T., Tominaga, S.: Estimation of Multiple Illuminants Based on Specular Highlight Detection. In: Schettini, R., Tominaga, S., Trémeau, A. (eds.) CCIW 2011. LNCS, vol. 6626, pp. 85-98. Springer, Heidelberg (2011) 